\title{
Pathological Analysis of Early Transplant Glomerulopathy in Renal Allografts Using Low-Vacuum Scanning Electron Microscopy
}

\author{
Hiroka Onishi ${ }^{a, b}$ Hideyo Oguchi ${ }^{b}$ Kazunobu Shinoda ${ }^{b}$ Tetuo Mikami $^{c}$

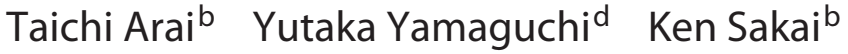 \\ ${ }^{a}$ Department of Nephrology, Toho University Graduate School of Medicine, Tokyo, Japan; ${ }^{b}$ Department of \\ Nephrology, Toho University Faculty of Medicine, Tokyo, Japan; 'Department of Pathology, Toho University \\ Faculty of Medicine, Tokyo, Japan; 'Yamaguchi's Pathology Laboratory, Chiba, Japan
}

\section{Keywords}

Antibody-mediated rejection - Glomerular basement membrane duplication - Kidney transplantation . Low-vacuum scanning electron microscopy - Transplant glomerulopathy

\begin{abstract}
Aim: Low-vacuum scanning electron microscopy (LVSEM) has been reported to aid in diagnosis of renal biopsy. This study evaluated early transplant glomerulopathy in kidney transplant recipients using LVSEM. Methods: We selected 4 biopsies of $c g 0,5$ biopsies of $c g 1 a, 5$ biopsies of $c g 1 b$, and 4 biopsies of cg2 lesions that had been evaluated by light microscopy (LM) and transmission electron microscopy from recipients with acute/active or chronic, active antibody-mediated rejection (AABMR or CAABMR). Renal allograft paraffin sections ( $1 \mu \mathrm{m}$ thickness) were stained with periodic acidmethenamine silver and observed using LVSEM. The cg score was based on the Banff classification. The parameter "percentage of duplicated capillary number" was calculated as follows: in 1 glomerulus with glomerular basement membrane (GBM) duplication, the total duplicated capillary number/the total number of capillaries $\times 100$. Results: In all 4 bi-
\end{abstract}

karger@karger.com www.karger.com/nef

Karger $\stackrel{\text { ' }}{=}$

GOPEN ACCESS
(C) 2020 The Author(s)

Published by S. Karger AG, Basel

This is an Open Access article licensed under the Creative Commons Attribution-NonCommercial-4.0 International License (CC BY-NC) (http://www.karger.com/Services/OpenAccessLicense), applicable to the online version of the article only. Usage and distribution for commercial purposes requires written permission. opsy specimens with AABMR showing cg0, LVSEM revealed GBM duplication not identified by $L M$. The average percentage of duplicated capillary number per glomerulus with GBM duplication was higher when observed by LVSEM than when observed by LM in all cg1 $\mathrm{b}$ and $\mathrm{cg} 2$ biopsy specimens. Conclusion: LVSEM revealed early GBM duplication in $A A B M R$. Early GBM duplication might progress in the very early phase of AABMR. GBM duplication was more frequently detected by LVSEM than by LM in biopsy specimens with early chronic, active antibody mediated rejection. Thus, LVSEM may be useful in diagnosis of early transplant glomerulopathy. (c) 2020 The Author(s)

Published by S. Karger AG, Basel

\section{Introduction}

The finding of transplant glomerulopathy has been reported as an independent risk factor for allograft failure in kidney transplant recipients [1]. In 2011, it was reported that early alterations in electron microscopy findings,

Hiroka Onishi and Hideyo Oguchi contributed equally to the present study. 
involving duplication of glomerular basement membrane (GBM), were found in recipients with antibody-mediated rejection early after kidney transplantation [2]. In that study, therapeutic intervention for antibody-mediated rejection with early glomerular alteration observed by electron microscopy was also shown to diminish the progression of overt transplant glomerulopathy [2]. The 2013 Banff meeting established a new score of cgla, which constitutes GBM duplication detected by electron microscopy, but not by light microscopy (LM) [3]. The Banff meeting report also noted that, worldwide, some transplant facilities do not have access to electron microscopy equipment [3].

In 2010, Inaga et al. [4] reported that spike lesions that were not clear by LM could be observed in periodic acidmethenamine silver-stained GBM using low-vacuum scanning electron microscopy (LVSEM). Subsequently, Okada et al. [5] reported that LVSEM could show irregular thickness and wrinkling of GBM on periodic acid-methenamine silver-stained specimens from patients with pediatric steroid-resistant nephrotic syndrome. The present study was performed to assess the early GBM duplication of antibody-mediated rejection using LVSEM.

\section{Materials and Methods}

\section{Patients}

In this study, the diagnostic criteria for acute/active antibodymediated rejection (AABMR) and chronic, active antibody-mediated rejection (CAABMR) were based on the Banff 2013 classification [3]. In the present study, diagnostic criteria for AABMR and CAABMR were defined only by histological findings according to Banff 2013 criteria [3] regardless of the presence of donor-specific antibody. The Banff 2013 criteria state that the cgla definition is the absence of GBM duplication by LM, but GBM duplication of at least 3 glomerular capillaries by electron microscopy [3]. In the present study, the absence of GBM duplication when observed by LM, but presence when observed by transmission electron microscopy (TEM), was classified in the cgla group; this classification was made regardless of the number of GBM duplications observed by TEM with modification of Banff 2013 criteria [3].

We extracted 9 biopsy specimens from 9 recipients of AABMR during routine pathological evaluation ( $\mathrm{cg} 0$ based on LM) from among 154 allograft biopsy specimens analyzed from January 2016 to December 2016 at the Toho University Omori Medical Center. Among the 9 biopsy specimens that were classified as cg0 when observed by LM, 5 biopsy specimens were classified as cgla when observed by LM and TEM; in these specimens, GBM duplication was not observed by LM, whereas it was observed by TEM. Among the 9 biopsy specimens that were classified $\operatorname{cg} 0$ when observed by LM, 4 biopsy specimens were classified as cg0 by LM and TEM; in these specimens, GBM duplication was not observed by LM and TEM. We also extracted 9 biopsy specimens from 8 recipients of CAABMR during routine pathological evaluation (cg1 or cg2, based on LM) from among 345 biopsy specimens analyzed from January 2016 to December 2017 at the same institution. We excluded biopsy specimens with 00 scores for AABMR and CAABMR, as well as biopsy specimens with $\mathrm{cg} 3$ scores for CAABMR. As controls, we included 2 biopsy specimens with IgA nephropathy and 2 biopsy specimens with minimal change disease.

\section{Histopathology}

The basic process of observation using LVSEM was performed in accordance with a previous report $[4,6]$ with modifications of paraffin section thickness, magnification of observation by LVSEM, and a portion of the observation process, as follows: briefly, separate from the biopsy specimens used for clinical diagnosis, $1-\mu \mathrm{m}$ paraffin sections were excised (instead of the $5-10-\mu \mathrm{m}$ sections reported previously [4]) to observe early transplant glomerulopathy, and they were deparaffinized for use in production of periodic acid-methenamine silver-stained specimens; photos of glomeruli of periodic acid-methenamine silver-stained sections were taken by LM, and cover glasses were then removed with xylene and specimens were observed using LVSEM (Hitachi Corporation TM-3030); regarding LVSEM observation, the glomerulus was observed at approximately $\times 500-1,800$ original magnification for detection of GBM duplication, and for suspicious findings for which GBM duplication could not be clearly determined at the same magnification, the glomerulus was observed at higher magnification (approximately $\times 1,800-7,000$ original magnification). In this study, pathological evaluation using LVSEM was performed by 2 nephrologists (H.O.1 and H.O.2), and we also consulted with 2 pathologists (T.M. and Y.Y.) about LVSEM observation.

TEM data were collected from images taken during routine clinical diagnosis. Regarding TEM observation in Toho Omori University, routine TEM observation images were taken at approximately $\times 1,000-3,000$ original magnification. The preparation of sections for TEM observation in Toho Omori University has been previously described [7]. In our study, different glomeruli were observed by TEM and LVSEM.

In the AABMR $(\operatorname{cg} 0)$ and CAABMR (cgla) biopsies where GBM duplication could not be observed by LM, numbers of duplicated glomeruli and duplicated capillaries were analysed by LVSEM or TEM. In the CAABMR biopsies (cg1b and cg2), we counted glomerular duplicated capillary number only in glomeruli where GBM duplication could be observed by LM.

The total count of glomerular capillary number with GBM duplication observed by LVSEM or LM, divided by total glomerular number, was also examined. This parameter is expressed as the average number of duplicated capillaries per glomerulus. Total glomerular number was evaluated by LVSEM. The parameter "percentage of duplicated capillary number" was calculated as follows: in 1 glomerulus with GBM duplication, the total duplicated capillary number/the total number of capillaries $\times 100$. The average percentage of duplicated capillary number per 1 glomerulus with GBM duplication was evaluated.

Regarding observations from biopsy specimens with CAABMR, mimicking Banff cg scores $[3,8]$, "GBM duplication score" was defined as follows: 0, no GBM duplication was observed in the glomerular capillary loops of 1 glomerulus; 1, GBM duplication was observed in 1-10\% of the glomerular capillary loops of 1 glomerulus; 2 , GBM duplication was observed in $10-25 \%$ of the glomerular capillary loops of 1 glomerulus; 3, GBM duplication was observed in $25-50 \%$ of the glomerular capillary loops of 1 glomerulus; 
Fig. 1. a LM image (original magnification, $\times 400)$ of 1 glomerulus in an AABMR biopsy specimen. b LVSEM image (original magnification, $\times 600$ ) of the glomerulus shown in panel a. c LM image of a glomerular capillary loop of the glomerulus shown in panel a (original magnification, $\times 400$ ). d LVSEM image (original magnification, $\times 3,000)$ of the glomerular capillary loop shown in panel c; panel d shows GBM duplication observed by LVSEM (arrow), but not by LM. e LM image of GBM duplication in a CAABMR (cg2) (original magnification, $\times 400$ ) biopsy specimen. $f$ LVSEM image (original magnification, $\times 2,000$ ) of GBM duplication (arrow) of the GBM shown in panel e. $\mathbf{g}$ TEM image of GBM duplication (arrow) in an AABMR (cgla by LM and TEM) biopsy specimen. h TEM image of GBM duplication (arrow) in a CAABMR (cg2 by LM) biopsy specimen. LM, light microscopy; AABMR, acute/active antibody-mediated rejection; LVSEM, Low-vacuum scanning electron microscopy; CAABMR, chronic, active antibodymediated rejection; GBM, glomerular basement membrane; TEM, transmission electron microscopy.
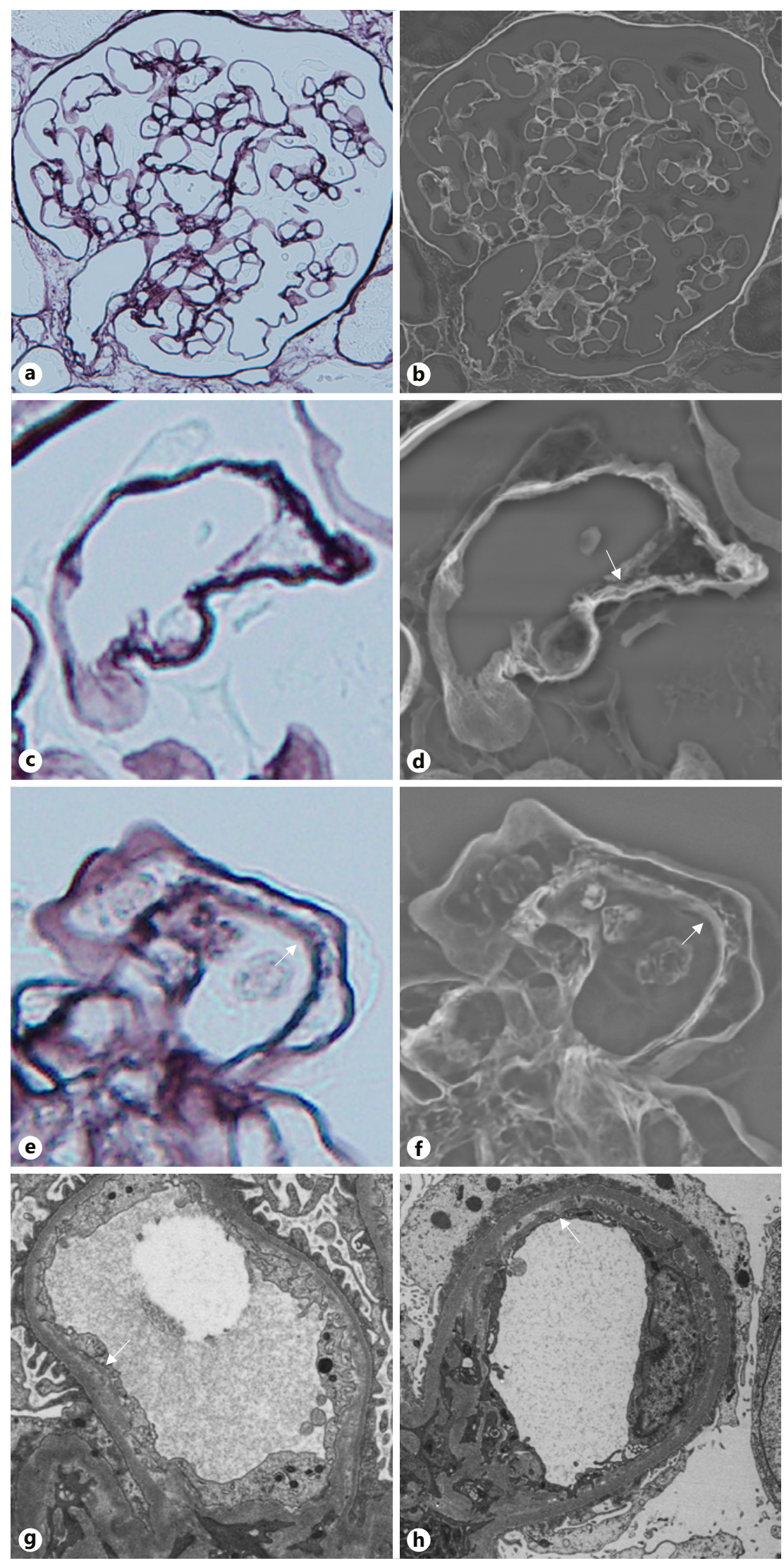
Table 1. Clinical background characteristics of biopsy specimens with AABMR and CAABMR

\begin{tabular}{lcccc}
\hline & $\begin{array}{c}\text { AABMR } \\
(\operatorname{cg} 0, n=4)\end{array}$ & $\begin{array}{l}\text { CAABMR } \\
(\operatorname{cg} 1 \mathrm{a}, n=5)\end{array}$ & $\begin{array}{c}\text { CAABMR } \\
(\operatorname{cg} 1 \mathrm{~b}, n=5)\end{array}$ & $\begin{array}{c}\text { CAABMR } \\
(\mathrm{cg} 2, n=4)\end{array}$ \\
\hline Male, $n$ & 3 & 4 & 2 & 2 \\
Duration after transplantation, months & $42.0 \pm 37.0$ & $14.4 \pm 14.1$ & $66.4 \pm 38.9$ & $91.8 \pm 59.4$ \\
Age of recipient at transplantation, years & $40.8 \pm 14.9$ & $39.2 \pm 15.0$ & $41.8 \pm 21.9$ & $44.0 \pm 14.8$ \\
Age of donor at transplantation, years & $57.0 \pm 5.0$ & $61.2 \pm 7.9$ & $56.8 \pm 13.0$ & $64.8 \pm 4.6$ \\
ABO incompatible, $n$ & 1 & 3 & 1 & 1 \\
2nd transplantation, $n$ & 1 & 1 & 1 & 0 \\
3rd transplantation, $n$ & 0 & 0 & 0 & 1 \\
Protocol biopsy, $n$ & 3 & 4 & 5 & 1 \\
Preexisting DSA, $n$ & 1 & 1 & 1 & 1 \\
De novo DSA, $n$ & 2 & $1.22 \pm 0.34$ & $1.87 \pm 0.65$ \\
Serum Cr, mg/dL & $1.67 \pm 1.13$ & $2.28 \pm 1.98$ & 1 & 1 \\
Treatment of diabetes, $n$ & 0 & 1 & 5 & 4 \\
Treatment of hypertension, $n$ & 3 & $1.20 \pm 0.45$ & $1.80 \pm 0.84$ & $1.75 \pm 0.50$ \\
g score & $1.25 \pm 0.50$ & $1.60 \pm 1.52$ & $1.40 \pm 1.34$ & $2.00 \pm 1.41$ \\
ptc score & $2.75 \pm 0.50$ & $2.20 \pm 1.10$ & $1.60 \pm 1.52$ & $1.50 \pm 1.73$ \\
c4d score & $1.50 \pm 1.29$ & & & 2 \\
\hline
\end{tabular}

Data shown are means \pm standard deviations. DSA, donor-specific antibody; AABMR, acute/active antibodymediated rejection; CAABMR, chronic, active antibody-mediated rejection.

4, GBM duplication was observed in $50-100 \%$ of the glomerular capillary loops of 1 glomerulus. Average GBM duplication scores were evaluated among glomeruli with GBM duplication by LM or LVSEM. Glomerular sclerosis and highly collapsed glomeruli were excluded from analysis in both AABMR and CAABMR biopsy specimens. In the present study, statistical comparisons were not performed because of the limited number of specimens.

\section{Results}

\section{Representative Figure of $A A B M R$ and $C A A B M R$}

Using LVSEM and TEM

Figure 1 shows representative images of biopsy specimens with AABMR and CAABMR, acquired using LM, LVSEM, and TEM. Figure 1 a shows 1 glomerulus from a sample with AABMR in an image (original magnification, $\times 400$ ) acquired via LM; Figure $1 \mathrm{~b}$ shows the same glomerulus in an image acquired via LVSEM (original magnification, $\times 600$ ). Figure $1 \mathrm{c}$ is an LM image of a glomerular capillary loop of the glomerulus shown in Figure $1 \mathrm{a}$; Figure $1 \mathrm{~d}$ is an LVSEM image (original magnification, $\times 3,000)$ of the glomerular capillary loop. Glomerular capillary duplication was observed by LVSEM, but not by LM (arrow in Fig. 1d). Figure 1e is an LM image of GBM duplication in a sample with CAABMR in an image acquired by LM (original magnification, $\times 400$ ); GBM duplication was clearly observed by LM (arrow in Fig. 1e). Figure if is an LVSEM image (original magnification, $\times 2,000$ ) of the GBM duplication shown in Figure 1e; GBM duplication was clearly observed by LVSEM (arrow in Fig. 1f). Figure $1 \mathrm{~g}$ is a TEM image of GBM duplication in a specimen from a sample with CAABMR (cg0 by LM, but cgla by TEM). Figure $1 \mathrm{~h}$ is a TEM image of GBM duplication in a sample with CAABMR (cg2 by LM). Figure $1 \mathrm{~g}$ and $\mathrm{h}$ both show GBM duplication observed by TEM (arrow in Fig. 1g, h).

\section{Clinicopathological Background Characteristics and Analysis of Biopsy Specimens with AABMR and CAABMR}

Table 1 shows the clinicopathological characteristics of biopsy specimens with AABMR (showing cg0 lesions), and CAABMR (showing cg1a, cg1b, and cg2 lesions). Table 2 shows the pathological evaluation of 4 biopsy specimens with AABMR (showing cg0 lesions) and 5 biopsy specimens with CAABMR (showing cgla lesions), as observed by LM and TEM. In all 4 biopsy specimens with cg0 lesions, glomeruli with GBM duplication were observed by LVSEM, but not by LM and TEM. The number of glomeruli observed by TEM was smaller than the number of glomeruli observed by LVSEM in each biopsy specimen except biopsy number 5 of cgla.

Tables 3 and 4 shows the pathological evaluation by LVSEM and TEM, with respect to cg1b and cg2 biopsy 
Table 2. Pathological characteristics of 9 biopsy specimens with antibody-mediated rejection in which GBM duplication was not observed by LM ( 4 biopsy specimens with AABMR show cg0 lesions and 5 biopsy specimens with CAABMR show cgla lesions)

\begin{tabular}{|c|c|c|c|c|c|c|c|}
\hline \multirow{2}{*}{$\begin{array}{l}\text { Biopsy } \\
\text { number }\end{array}$} & \multicolumn{4}{|l|}{ LVSEM } & \multicolumn{3}{|l|}{ TEM } \\
\hline & $\begin{array}{l}\text { total number } \\
\text { of glomeruli }\end{array}$ & $\begin{array}{l}\text { number of duplicated } \\
\text { glomeruli number (number } \\
\text { of duplicated capillaries) }\end{array}$ & $\begin{array}{l}\text { average number of } \\
\text { duplicated capillary } \\
\text { per } 1 \text { glomerulus }\end{array}$ & $\begin{array}{l}\text { average percentage of } \\
\text { duplicated capillary number } \\
\text { per } 1 \text { duplicated glomerulus }\end{array}$ & $\begin{array}{l}\text { number of } \\
\text { glomeruli } \\
\text { observed }\end{array}$ & $\begin{array}{l}\text { number of duplicated } \\
\text { glomeruli (number of } \\
\text { duplicated capillaries) }\end{array}$ & $\begin{array}{l}\text { density of duplicated } \\
\text { caplillary per observed area } \\
\left(\text { number } \times 10^{-4} / \mu \mathrm{m}^{2}\right)\end{array}$ \\
\hline \multicolumn{8}{|l|}{$\operatorname{cgO}$} \\
\hline 1 & 15 & $4(4)$ & 0.27 & 2.19 & 1 & $0(0)$ & 0 \\
\hline 2 & 27 & $5(7)$ & 0.26 & 2.72 & 2 & $0(0)$ & 0 \\
\hline 3 & 27 & $2(2)$ & 0.07 & 2.00 & 3 & $0(0)$ & 0 \\
\hline 4 & 13 & $1(1)$ & 0.08 & 3.23 & 1 & $0(0)$ & 0 \\
\hline \multicolumn{8}{|l|}{$\operatorname{cgla}$} \\
\hline 1 & 11 & $1(1)$ & 0.09 & 5.00 & 1 & $1(2)$ & 0.3 \\
\hline 2 & 24 & $3(3)$ & 0.13 & 1.68 & 1 & $1(3)$ & 2.3 \\
\hline 3 & 24 & $2(3)$ & 0.13 & 3.43 & 2 & $2(2)$ & 1.2 \\
\hline 4 & 7 & $1(1)$ & 0.14 & 1.61 & 4 & $4(5)$ & 1.8 \\
\hline 5 & 4 & $3(3)$ & 0.75 & 6.74 & 4 & $1(6)$ & 0.8 \\
\hline
\end{tabular}

DSA, donor-specific antibody; AABMR, acute/active antibody-mediated rejection; CAABMR, chronic, active antibody-mediated rejection; GBM, glomerular basement membrane; LVSEM, low-vacuum scanning electron microscopy; TEM, transmission electron microscopy; LM, light microscopy.

Table 3. Pathological characteristics assessed by LVSEM of 5 biopsy specimens showing cglb lesions and 4 biopsy specimens showing cg2 lesions

\begin{tabular}{|c|c|c|c|c|c|c|c|c|}
\hline $\begin{array}{l}\text { Biopsy } \\
\text { number }\end{array}$ & $\begin{array}{l}\text { Total number } \\
\text { of glomeruli } \\
\text { by LVSEM }\end{array}$ & $\begin{array}{l}\text { Number of } \\
\text { duplicated } \\
\text { glomeruli by } \\
\text { LM and LVSEM }^{*}\end{array}$ & $\begin{array}{l}\text { Average number of } \\
\text { duplicated capillary by } \\
\text { LM per } 1 \text { glomerulus }\end{array}$ & $\begin{array}{l}\text { Average number of } \\
\text { duplicated capillary } \\
\text { by LVSEM per } \\
1 \text { glomerulus }\end{array}$ & $\begin{array}{l}\text { Average percentage of } \\
\text { duplicated capillary } \\
\text { number per } 1 \text { duplicated } \\
\text { glomerulus by LM }\end{array}$ & $\begin{array}{l}\text { Average percentage of } \\
\text { duplicated capillary } \\
\text { number per } 1 \text { duplicated } \\
\text { glomerulus by LVSEM }\end{array}$ & $\begin{array}{l}\text { Average GBM } \\
\text { duplication score } \\
\text { among the duplicated } \\
\text { glomeruli by LM }\end{array}$ & $\begin{array}{l}\text { Average GBM } \\
\text { duplication score } \\
\text { among the duplicated } \\
\text { glomeruli by LVSEM }\end{array}$ \\
\hline 1 & 41 & 4 & 0.10 & 0.27 & 2.07 & 5.68 & 1.00 & 1.25 \\
\hline 2 & 8 & 2 & 0.38 & 0.50 & 2.36 & 3.50 & 1.00 & 1.00 \\
\hline 3 & 5 & 3 & 1.60 & 2.80 & 4.82 & 7.60 & 2.00 & 3.00 \\
\hline \multicolumn{9}{|l|}{$c g 2$} \\
\hline 1 & 11 & 3 & 0.73 & 1.00 & 5.01 & 6.40 & 1.67 & 1.67 \\
\hline 2 & 6 & 3 & 1.67 & 2.17 & 3.43 & 4.48 & 1.67 & 1.67 \\
\hline 3 & 32 & 13 & 2.06 & 2.41 & 13.00 & 14.95 & 2.23 & 2.38 \\
\hline 4 & 24 & 8 & 1.54 & 1.75 & 8.60 & 9.65 & 2.25 & 2.25 \\
\hline
\end{tabular}

LVSEM, low-vacuum scanning electron microscopy; LM, light microscopy. * Glomerular duplicated capillary number was counted only in glomeruli where GBM duplication could be observed by LM in the CAABMR biopsies (cg1b and cg2).

specimens. When the average number of duplicated capillaries per glomerulus was evaluated, the values observed by LVSEM were higher than those observed by LM in all cglb and cg2 biopsy specimens. When the average percentage of duplicated capillary number per duplicated glomerulus was evaluated, the values observed by LVSEM were also higher than those observed by LM in all cglb and cg2 biopsy specimens. When the average GBM du- plication score among duplicated glomeruli was evaluated, the values observed by LVSEM were higher than those observed by LM in 4 of 5 cglb biopsy specimens and 1 of 4 cg2 biopsy specimens.

As controls, we analyzed 2 specimens with IgA nephropathy and 2 specimens with minimal change disease. GBM duplication was not evident observed by LVSEM or by TEM in any of these 4 controls. 
Table 4. Pathological characteristics assessed by TEM of 5 biopsy specimens showing cglb lesions and 4 biopsy specimens showing cg2 lesions

\begin{tabular}{lllll}
\hline $\begin{array}{l}\text { Biopsy } \\
\text { number }\end{array}$ & $\begin{array}{l}\text { Number of } \\
\text { glomeruli ob- } \\
\text { served by TEM }\end{array}$ & $\begin{array}{l}\text { Number of dupli- } \\
\text { cated glomeruli } \\
\text { observed by TEM }\end{array}$ & $\begin{array}{l}\text { Total glomerular capil- } \\
\text { lary number of GBM } \\
\text { duplication by TEM }\end{array}$ & $\begin{array}{l}\text { Density of duplicated } \\
\text { capillary per observed } \\
\left.\text { area (number } \times 10^{-4} / \mu m^{2}\right)\end{array}$ \\
\hline cg1b & & & & \\
1 & 2 & 1 & 3 & 1.0 \\
2 & 1 & 1 & 10 & 4.0 \\
3 & 1 & 1 & 5 & 1.0 \\
4 & 3 & 3 & 37 & 15.0 \\
5 & 1 & 1 & 2 & 2.0 \\
\hline$c g 2$ & & & & \\
1 & 1 & 1 & 15 & 12.0 \\
2 & 1 & 0 & 0 & 0.0 \\
3 & 1 & 1 & 18 & 8.0 \\
4 & 2 & 2 & 40 & 21.0 \\
\hline
\end{tabular}

TEM, transmission electron microscopy.

\section{Discussion}

To the best of our knowledge, analyses of transplant glomerulopathy using LVSEM have rarely been reported. In 2020, Yokoyama et al. [9] reported the possibility of detection for initial GBM change of antibody-mediated rejection in kidney allograft biopsies assessed by LVSEM. The aim of the present study was to identify characteristics of early transplant glomerulopathy that could be observed by LVSEM, but not by LM.

A previous study showed that observations using electron microscopy result in a rate of chronic rejection diagnosis two-fold higher than that of observations using LM [10]. In the present study, we used LVSEM and TEM to analyze biopsy specimens with early transplant glomerulopathy. Inaga et al. [4] reported that renal biopsy paraffin specimens with 5- to 10- $\mu \mathrm{m}$ thickness were prepared for observation by LVSEM. A recent study in 2020 also has prepared specimens of paraffin biopsy with $5-\mu \mathrm{m}$ thickness to observe GBM change in antibody-mediated rejection biopsy samples assessed by LVSEM [9]. The present study aimed to use LVSEM to detect early transplant glomerulopathy that could not be observed by LM. Therefore, we presumed that it would be more feasible to observe GBM duplication in the early stage of transplant glomerulopathy by using thinner sections; accordingly, we observed the specimens by using $1-\mu \mathrm{m}$ sections. We presumed that this approach might enable observation of GBM duplication in early stages of trans- plant glomerulopathy, which could not be observed by LM. Furthermore, by using $1-\mu \mathrm{m}$ sections, we were able to observe the GBM of the same glomerulus using both LM and LVSEM. Previously, Inaga et al. [6] showed that observations of cells and tissues with platina blue stain at high magnification (to $\times 10,000$ magnification) could be performed by LVSEM. In our study, we observed $1-\mu \mathrm{m}$ periodic acid-methenamine silver-stained sections at a maximum magnification of approximately $\times 7,000$; we presumed that this might enable detection of GBM duplication in biopsy specimens with early transplant glomerulopathy.

LVSEM revealed GBM duplication in 4 biopsy specimens with AABMR showing $\operatorname{cg} 0$, when evaluated by LVSEM and TEM (Table 2). These results indicate that none of the biopsy specimens were classified as AABMR, when evaluated by LVSEM, in the present study. All cg0 biopsy specimens evaluated by LM revealed GBM duplication assessed by LVSEM, and this might be influenced by the overestimation or artifact. Although the number of specimens was quite small, which limits the strength of the conclusions and might be overestimated, early transplant glomerulopathy lesions might be observed more frequently during early stages of AABMR. Greater numbers of glomeruli could be observed by LVSEM than by TEM. GBM duplication varied depending on the sampling area in sections observed by TEM. In the 4 biopsy specimens with cg0 in Table 2, GBM duplication was not observed by TEM and could be observed LVSEM; this was presumably affected by glomerular sampling.

Using TEM, many glomerular duplicated capillaries could be observed in some biopsies of cg1b and cg2 (Table 4). We presumed that very early GBM duplication would be easy to observe by TEM because ultra-thin sections are used for TEM observation (approximately 70-nm sections are used for TEM observation in Toho Omori University). The differences in section thickness between LVSEM and TEM observations might have affected the very low rate of detection of GBM duplication in early lesions of AABMR (cg0) or CAABMR (cgla) by LVSEM (Table 2). In addition, 3 of 4 biopsy specimens with AABMR (cg0) and 4 of 5 biopsy specimens with CAABMR (cg1a) were subclinical antibody-mediated rejection diagnosed by protocol biopsy. Furthermore, average $g$ scores were low in biopsy specimens with AABMR and CAABMR (Table 1). Very early and mild lesions in recipients with antibody-mediated rejection may affect very low detection rate of early GBM duplication.

In all biopsy specimens with CAABMR (cg1b and cg2 by LM), the average number of duplicated capillaries per
76

Nephron 2020;144(suppl 1):71-78 DOI: $10.1159 / 000512136$
Onishi/Oguchi/Shinoda/Mikami/Arai/ Yamaguchi/Sakai 
glomerulus and average percentage of duplicated capillary number per duplicated glomerulus were higher when observed by LVSEM than when observed by LM (Table 3). These findings indicate that the sensitivity in detection of GBM duplication may be higher when observed by LVSEM than when observed by LM in biopsy specimens with early CAABMR. Regarding the evaluation of GBM duplication score, the values observed by LVSEM were higher than those observed by LM in 4 of 5 cg $1 \mathrm{~b}$ specimens and 1 of $4 \mathrm{cg} 2$ specimens. The difference in average percentage of duplicated capillary number per duplicated glomerulus was small, when compared between LVSEM and LM observation (Table 3). This degree of difference is unlikely to occur when using a semiquantitative score, such as the GBM duplication score.

The major limitation of this study is that different glomeruli were observed by LVSEM and TEM. Therefore, we could not clearly determine whether TEM or LVSEM exhibited superior diagnostic sensitivity with respect to GBM duplication not observed by LM in a single glomerulus. Second, a previous report showed that alterations of glomerular endothelial cells were observed in the subendothelial area in biopsies of transplant glomerulopathy [11]; however, our assessments of endothelial cell damage were limited to analyses of GBM duplication. Third, the number of samples was quite small in this study; thus, statistical comparison could not be performed on the paired data. Accordingly, further studies are needed to fully elucidate the relationship between early transplant glomerulopathy diagnosed by LVSEM and the clinical prognosis of affected recipients.

In conclusion, we could detect early GBM duplication as an early chronic lesion that could be observed by LVSEM, but not observed by LM in AABMR biopsy specimens. Early GBM duplication may develop in the very early phase of AABMR. LVSEM also revealed more GBM duplication, compared with LM, in the early phase of CAABMR. Therefore, LVSEM may be a useful tool for the diagnosis of early lesions in kidney transplant recipients with antibody-mediated rejection.

\section{Acknowledgements}

We thank the kidney transplantation team, Department of $\mathrm{Ne}$ phrology, Toho University Faculty of Medicine, for contribution to execution or planning or design of the study. We thank Dr. Kazutoshi Shibuya, Department of Surgical Pathology, Toho University Faculty of Medicine, for contribution to managing the preparation of biopsy specimens. We thank Professor Kazuho Honda in the Department of Anatomy, Showa University School of Medicine, and Ms. Mayumi Ishii of the Department of Surgical Pathology, Toho University Faculty of Medicine, for helpful comments regarding our study. We thank Ryan Chastain-Gross, $\mathrm{PhD}$, from Edanz Group (https://en-author-services.edanzgroup.com) for editing the draft of this manuscript.

\section{Statement of Ethics}

This study protocol was approved by Toho University Omori Medical Center Ethics Committee (approval number: M-19061) and implemented as grant research by the LVSEM study group.

\section{Conflict of Interest Statement}

The authors have no conflict of interest regarding the present study.

\section{Author Contibutions}

H. Onishi and H. Oguchi designed this study, collected cliniopathological data, and wrote the paper. K. Shinoda contributed to clinical data acquisition and participated in drafting the work. T.M. and Y.Y. contributed to pathological evaluation and participated in drafting the work. T.A. collected in clinical data and participated in drafting the work. K. Sakai contributed the study design and participated in drafting the work.

\section{References}

LVSEM Analysis of Transplant Glomerulopathy
1 Gloor JM, Sethi S, Stegall MD, Park WD, Moore SB, DeGoey S, et al. Transplant glomerulopathy: subclinical incidence and association with alloantibody. Am J Transplant. 2007;7(9):2124-32.

2 Haas M, Mirocha J. Early ultrastructural changes in renal allografts: correlation with antibody-mediated rejection and transplant glomerulopathy. Am J Transplant. 2011; 11(10):2123-31.
3 Haas M, Sis B, Racusen LC, Solez K, Glotz D, Colvin RB, et al. Banff 2013 meeting report: inclusion of c4d-negative antibody-mediated rejection and antibody-associated arterial lesions. Am J Transplant. 2014;14(2):272-83.

4 Inaga S, Kato M, Hirashima S, Munemura C, Okada S, Kameie T, et al. Rapid three-dimensional analysis of renal biopsy sections by low vacuum scanning electron microscopy. Arch Histol Cytol. 2010;73(3):113-25. 
5 Okada S, Inaga S, Kawaba Y, Hanada T, Hayashi A, Nakane H, et al. A novel approach to the histological diagnosis of pediatric nephrotic syndrome by low vacuum scanning electron microscopy. Biomed Res. 2014; 35(4):227-36.

6 Inaga S, Hirashima S, Tanaka K, Katsumoto T, Kameie T, Nakane H, et al. Low vacuum scanning electron microscopy for paraffin sections utilizing the differential stainability of cells and tissues with platinum blue. Arch Histol Cytol. 2009;72(2):101-6.
7 Kawaguchi Y, Oguchi H, Mikami T, Yamaguchi Y, Kawamura T, Muramatsu M, et al. Donor-recipient body weight mismatch may affect glomerular basement membrane thinning in electron microscopic examination of 1-h renal allograft biopsy specimens. Transplant Proc. 2019;51(5):1348-52.

8 Racusen LC, Solez K, Colvin RB, Bonsib SM, Castro MC, Cavallo T, et al. The Banff 97 working classification of renal allograft pathology. Kidney Int. 1999;55(2):713-23.

9 Yokoyama H, Okada S, Yamada Y, Kitamoto $\mathrm{K}$, Inaga $\mathrm{S}$, Nakane $\mathrm{H}$, et al. Low-vacuum scanning electron microscopy may allow early diagnosis of human renal transplant antibody-mediated rejection. Biomed Res. 2020; 41(2):81-90.
10 Ivanyi B, Kemeny E, Szederkenyi E, Marofka F, Szenohradszky P. The value of electron microscopy in the diagnosis of chronic renal allograft rejection. Mod Pathol. 2001;14(12): $1200-8$

11 Wavamunno MD, O'Connell PJ, Vitalone M, Fung CL, Allen RD, Chapman JR, et al. Transplant glomerulopathy: ultrastructural abnormalities occur early in longitudinal analysis of protocol biopsies. Am J Transplant. 2007; 7(12):2757-68. 